\title{
Cytotoxic, chemosensitizing and radiosensitizing effects of curcumin based on thioredoxin system inhibition in breast cancer cells: 2D vs. 3D cell culture system
}

\author{
SHAYMAA ESSAM EL FEKY ${ }^{1 *}$, MAGDA ABDEL GHANY MEGAHED ${ }^{2}$, NADIA AHMED ABD EL MONEIM ${ }^{3}$, \\ EBTSAM RIZQ ZAHER ${ }^{1}$, SHADWA AHMED KHAMIS ${ }^{2}$ and LAMIAA MOHAMED AHMED ALI ${ }^{2 *}$ \\ Departments of ${ }^{1}$ Radiation Sciences, ${ }^{2}$ Biochemistry and ${ }^{3}$ Cancer Management and Research, \\ Medical Research Institute, University of Alexandria, Alexandra 21561, Egypt
}

Received January 25, 2020; Accepted October 15, 2020

DOI: $10.3892 /$ etm.2021.9937

\begin{abstract}
Targeting the thioredoxin/thioredoxin reductase ( $\operatorname{Trx} / \operatorname{TrxR}$ ) system is a promising strategy to overcome cancer resistance to conventional therapy. The present study investigated the effect of curcumin on the Trx/TrxR system either alone or in combination with chemotherapy, or radiotherapy in human MCF-7 breast cancer cells seeded in 2 and 3D culture systems. Cell viability, thioredoxin reductase 1 (TrxR1) activity, and the genetic expression of Trx, TrxR1, Bcl2 and BAX genes were studied. The findings showed that the mode of culture significantly affected the response of cancer cells to different treatment modalities, as well as their gene expression patterns. Curcumin treatment resulted in a reduction of breast cancer cell proliferation and induction of apoptosis, an effect that may be mediated by manipulating Trx system components, mainly Trx expression, and to a lesser extent TrxR1 expression and concentration. Furthermore, curcumin increased the sensitivity of breast cancer cells to chemotherapy and radiotherapy by reducing Trx and TrxR1 expression levels. Thus, curcumin may have a potential role as a dose-modifying agent that can be used either to sensitize resistant cells to therapy or to reduce the dose of these therapeutic agents.
\end{abstract}

\section{Introduction}

Breast cancer is the most common type of cancer diagnosed in women and ranked as the fifth leading cause of death (1). Cancer cells have an elevated level of reactive oxygen species (ROS) compared with healthy cells, and exhibit

Correspondence to: Dr Shaymaa Essam El Feky, Department of Radiation Sciences, Medical Research Institute, University of Alexandria, 165 Hurreya Avenue, Alexandria 21561, Egypt

E-mail: shayma.elfeky@gmail.com

\section{*Contributed equally}

Key words: chemosensitizer, curcumin, radiosensitizer, thioredoxin, thioredoxin reductase $1,3 \mathrm{D}$ cultures uncontrolled proliferative and high metabolic natures (2). This elevated ROS level is essential to maintain a cancer phenotype and makes cancer cells more susceptible to oxidative stress. Consequently, cancer cells resist this effect by increasing antioxidant levels, including that in the thioredoxin/thioredoxin reductase ( $\operatorname{Tr} x / \operatorname{Trx} R)$ system (3).

The antioxidant function of $\operatorname{Tr} / \operatorname{Trx} R$ system is not only beneficial for healthy cells, but also for cancer cell development as it has anti-apoptotic and angiogenesis functions (4). In addition, it has been reported that a high level of $\operatorname{Trx} / \operatorname{Trx} R$ increases cancer resistance to conventional treatment, such as chemotherapy and radiotherapy (5-7). Therefore, the last few years have witnessed an increasing interest towards inhibiting the Trx/TrxR system by natural or synthetic molecules, including the natural electrophilic inhibitor curcumin (8).

Curcumin, a natural polyphenol extracted from turmeric, has several therapeutic potentials due to its antioxidant and anti-inflammatory activities. Various studies have reported its tumor suppressive role via suppression of cellular proliferation, induction of apoptosis and irreversible inhibition of the Trx/TrxR system (9-11). Furthermore, few reports have demonstrated the role of curcumin in increasing tumor cell sensitivity to radiotherapy and cisplatin in vitro using $2 \mathrm{D}$ monolayer culture $(12,13)$.

Although the traditional 2D cell culture is an established system for cell-based studies, it does not reflect the real cellular behavior in vivo. Cells grown in monolayers differ in their cellular and extracellular interactions, which leads to altered morphologies, proliferative behaviors, genetic expression levels, treatment responses, and impaired access to metabolites and extracellular signals. As a result, the data can be misleading regarding in vivo responses $(14,15)$. Using a 3D cell culture system overcomes the disadvantages of a 2D system, as it represents a microenvironment similar to that in vivo (16). Numerous publications have focused on developing 3D models of the MCF-7 cell line to gain a better understanding of breast cancer biology and overcome the limitations associated with the traditional 2D models $(17,18)$.

Herein, the present study focused on studying the effect of curcumin on the Trx/TrxR system in breast cancer cells either alone or in combination with radio- or chemotherapy using 2 and $3 \mathrm{D}$ culture systems. 


\section{Materials and methods}

Cell culture. The human breast adenocarcinoma MCF-7 cell line (ATCC HTB-22 ${ }^{\mathrm{TM}}$ ) was maintained in DMEM/F12 (Lonza Group, Ltd.) supplemented with $10 \%$ fetal bovine serum (Sigma-Aldrich; Merck KGaA), $100 \mathrm{IU} / \mathrm{ml}$ penicillin and $100 \mathrm{mg} / \mathrm{ml}$ streptomycin. Cells were cultures in a humidified atmosphere at $37^{\circ} \mathrm{C}$ with $5 \% \mathrm{CO}_{2}$.

For the 2D monolayer culture, cells continued to be cultured in 6- and 96-well tissue culture plates as aforementioned. Cells were examined under an inverted microscope after 48 h. MCF-7 cells formed a confluent adherent layer (70-80\% confluence). After completion of the treatment protocols listed below, MCF-7 monolayer 2D cells were harvested using $0.05 \%$ trypsin and $0.02 \%$ EDTA.

For the 3D spheroid cultures, 96-well plates were pre-coated with $1.5 \%$ agarose and allowed to solidify for at least $2 \mathrm{~h}$ before loading the cells. MCF-7 cells were seeded in the pre-coated wells at a density of $1 \times 10^{5}$ cells/well and the plates were incubated in a humidified atmosphere with $5 \%$ $\mathrm{CO}_{2}$ at $37^{\circ} \mathrm{C}$. The culture medium was changed every 2 days until spheroids were formed. Spheroids were examined using a light microscope (magnification, x40) and digital camera (Carl Zeiss AG).

Treatments of MCF-7 cells. MCF-7 cells cultured in 2D monolayers and 3D spheroids were treated with the following: i) Curcumin (Loba Chemie Pvt. Ltd.) at concentrations of $10,20,30,50$ and $100 \mu \mathrm{M}$ for 24 and $48 \mathrm{~h}$; ii) 5-fluorouracil (5-FU)-Adriamycin-cyclophosphamide (FAC)-based chemotherapy $(22.5 \mu \mathrm{g} / \mathrm{ml}$ 5-fluorouracil, $0.579 \mu \mathrm{M}$ Adriamycin and $3 \mu \mathrm{g} / \mathrm{ml}$ cyclophosphamide) either alone or in combination with various curcumin concentrations for 24,48 and $72 \mathrm{~h}$; or iii) radiation whereby cells were exposed to irradiation at room temperature using a PRIMUSTM linear accelerator (Siemens AG) at a dose of $200 \mathrm{MU} / \mathrm{min}$ for the time required to apply doses of 2, 4, 6 and $8 \mathrm{~Gy}$. Radiation treatment was applied on untreated cells and curcumin-treated cells. Control cells were treated with DMSO.

Cell viability assay. The viability of MCF-7 cells was evaluated using an MTT assay. After treatment, cells were incubated with $0.5 \mathrm{mg} / \mathrm{ml}$ of MTT reagent for $3 \mathrm{~h}$ at $37^{\circ} \mathrm{C}$. After the MTT incubation, the MTT/medium was removed, and the precipitated crystals were dissolved in ethanol/DMSO (1:1) solution with agitation for $20 \mathrm{~min}$. The absorbance was measured at $540 \mathrm{~nm}$.

Measurement of TrxR1 concentration. TrxR1 concentration was determined using a Human TrxR1 ELISA kit (cat. no. In-Hu4061; Innova Biotech Co. Ltd.). Following treatment, cells were washed with PBS, trypsinized, and centrifuged at $24 \mathrm{x} \mathrm{g}$ for $5 \mathrm{~min}$ at $4^{\circ} \mathrm{C}$. The cells were lyzed by repeated freezing/thawing cycles, and then the lysate was centrifuged for $15 \mathrm{~min}$ at $18,600 \mathrm{x} \mathrm{g}$ at $4^{\circ} \mathrm{C}$. The supernatant was collected for protein and activity measurements. Total protein measurement was performing using the standard Lowry method (19). For TrxR1 concentration measurements, samples were processed according to the manufacturer's protocol. The TrxR1 content is expressed as $\operatorname{Tr} x \mathrm{R} 1 /$ protein $(\mathrm{pg} / \mathrm{mg})$.
Reverse transcription-quantitative polymerase chain reaction $(R T-q P C R)$. The gene expression of $\operatorname{Trx}, \operatorname{Trx} \mathrm{R} 1, \mathrm{Bcl} 2$ and BAX was determined using RT-qPCR. Total cellular RNA of monolayer cells and spheroids were extracted using a QIAamp RNA Blood Mini kit (Qiagen, Inc.) according to the manufacturer's instructions. Equal amounts of RNA were reverse transcribed using a miScript II reverse transcription kit (Qiagen, Inc.). The RNA samples were incubated with reverse transcription mixture at $37^{\circ} \mathrm{C}$ for $60 \mathrm{~min}$ followed by $5 \mathrm{~min}$ incubation at $95^{\circ} \mathrm{C}$. Subsequently, the RT-qPCR reaction was performed on an Applied Biosystems Real-Time PCR system using QuantiTecht SYBR Green RT-PCR Master mix (Qiagen, Inc.). The primer sequences were as follows: Trx forward, 5'-CGAGTCTTGAAGCTCTGTTTGG-3' and reverse, 5'-TAT CACCTGCAGCGTCCAAG-3'; TrxR1 forward, 5'-CTCAAA TTCTTGCTTATCAGGAGGG-3' and reverse, 5'-GCGACA TAGGATGCTCCAACA-3'; Bcl2 forward, 5'-ACAGGGTAC GATAACCGGGA-3' and reverse, 5'-GCCCAGACTCACATC ACCAA-3'; BAX forward, 5'-CCCTTTTGCTTCAGGGGA TGAT-3' and reverse, 5'-GGCGTCCCAAAGTAGGAGAG 3'; GAPDH forward, 5'-CCACATCGCTCAGACACCAT-3' and reverse, 5' AGCCAAATTCGTTGTCATACTTCT 3'. The RT-qPCR cycling conditions were as follows: An initial activation step of $15 \mathrm{~min}$ at $95^{\circ} \mathrm{C} ; 45$ cycles of $15 \mathrm{sec}$ at $94^{\circ} \mathrm{C}$, $30 \mathrm{sec}$ at $59^{\circ} \mathrm{C}$ and $30 \mathrm{sec}$ at $70^{\circ} \mathrm{C}$. Results are presented as the average fold change of target gene in test to control group using the $2^{-\Delta \Delta \mathrm{Cq}}$ formula (20).

Statistical analysis. Data were analyzed using SPSS software version 20.0. (IBM Corp.). Normally distributed quantitative data are expressed as the mean \pm standard deviation. Student's t-tests were used for comparing two variables or two groups and the F-test (ANOVA) was used to compare $>$ two groups with Tukey's post hoc test for pairwise comparisons. $\mathrm{P}<0.05$ was considered to indicate a statistically significant difference.

\section{Results}

Cell viability. MCF-7 cells cultured as 2D monolayer and 3D spheroids were treated with different curcumin concentrations $(10,20,30,50$ and $100 \mu \mathrm{M})$, cell viability was determined after 24 and $48 \mathrm{~h}$ of treatment. As demonstrated in Fig. 1, time- and concentration-dependent cytotoxic effects were observed in the 2 and 3D cultures. A significant amount of cell death $(\mathrm{P}<0.001)$ was observed in both cultures compared with the control at $30 \mu \mathrm{M}$ concentration and above, with a more pronounced effect after $48 \mathrm{~h}$ of treatment. Furthermore, a significant difference $(\mathrm{P}<0.001)$ was observed between the 2 and $3 \mathrm{D}$ cultures at $30 \mu \mathrm{M}$ concentrations and above. After $48 \mathrm{~h}$ treatment, results showed that $2 \mathrm{D}$ cultures were more sensitive to the cytotoxic effect of curcumin compared with the 3D cultures, cell viability percentages were 61,57 and $30 \%$ for the 2D monolayer, and 79,63 and 45\% for the 3D spheroids at 30, 50 and $100 \mu \mathrm{M}$ concentrations, respectively (Fig. 1).

A time-dependent cytotoxic effect was observed in cells treated with chemotherapy compared with the control group, with significantly reduced viability in the $2 \mathrm{D}$ cultures compared with that in the $3 \mathrm{D}$ cultures $(\mathrm{P}<0.001)$. Cell viability percentages were 65 and $44 \%$ for the 2D monolayer, and 98 and $91 \%$ for the 3D spheroids after 24 and 48 of treatment, 


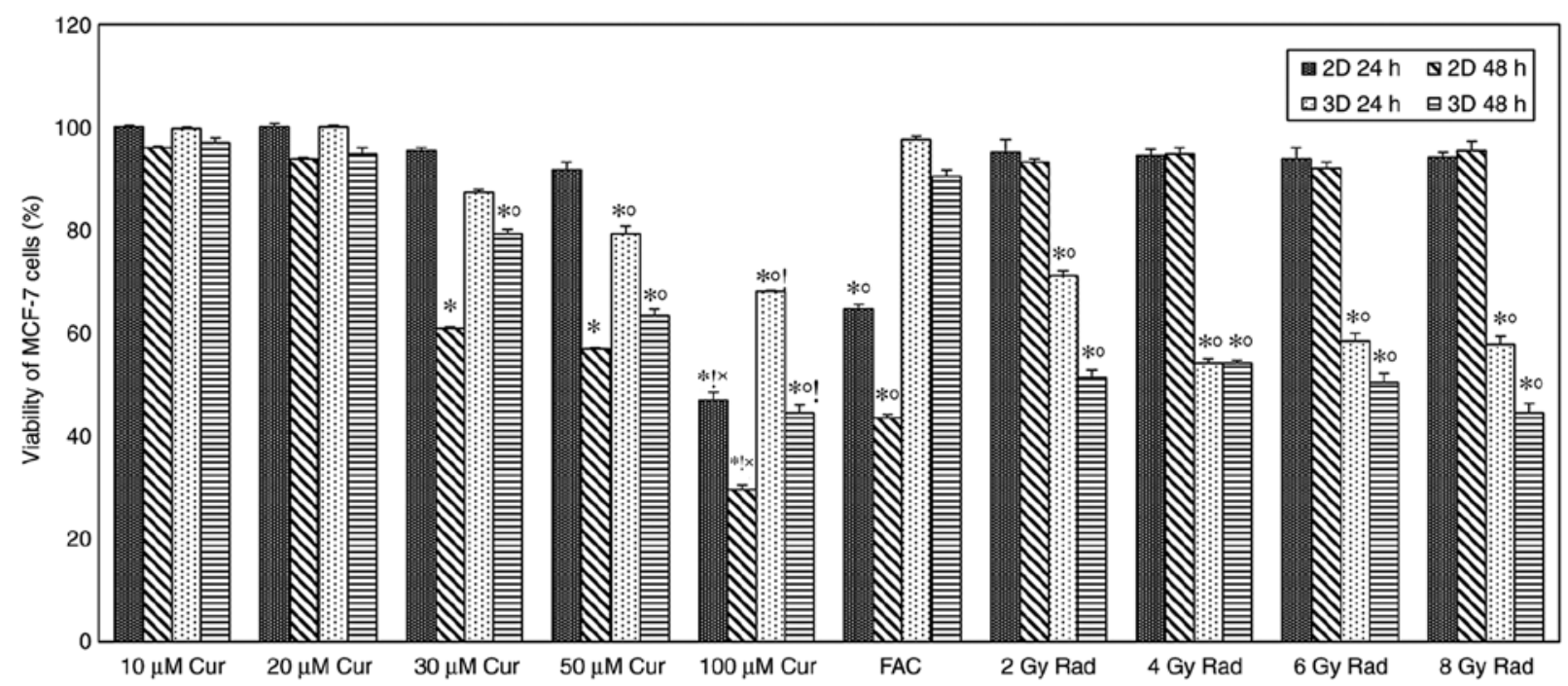

Figure 1. Viability of MCF-7 cells under different conditions. MCF-7 cells were treated with different concentrations of curcumin, FAC and radiation $(2,4$, 6 and $10 \mathrm{~Gy})$. Results represent the \% relative to the control and are presented as mean $\pm \mathrm{SD}(\mathrm{n}=5)$. ${ }^{*} \mathrm{P}<0.001$ vs. $100 \%$ viability of untreated cells; ${ }^{\circ} \mathrm{P}<0.001$ $2 \mathrm{D}$ vs. $3 \mathrm{D}$ culture; $\mathrm{P}<0.001$ vs. FAC; and ${ }^{\times} \mathrm{P}<0.001$ vs. all doses of Rad. Cur, curcumin; FAC, 5 -Fluorouracil-Adriamycin-cyclophosphamide; Rad, radiation.

respectively (Fig. 1). Exposing cells to different doses of ionizing radiation showed a slight amount of cell death in the 2D monolayer, while in the 3D spheroid group, cell death was significantly increased compared with the control $(\mathrm{P}<0.001$ for all doses; Fig. 1).

Comparing the effect of curcumin treatment on cell viability with standard treatments did not show any significant difference at low doses; however, the viability of 2D cells treated with $100 \mu \mathrm{M}$ curcumin was significantly lower compared with those treated with FAC and radiation. In the 3D model, $100 \mu \mathrm{M}$ curcumin-treated cells had significantly lower viability compared with FAC, but not with radiation (Fig. 1).

When chemotherapy was combined with curcumin, cell viability was significantly reduced with increasing curcumin concentration and treatment duration in 2 and $3 \mathrm{D}$ cultures ( $\mathrm{P}<0.001$ for all comparisons). A significantly higher rate of cell death was observed in 2D cultures compared with 3D cultures at all-time intervals $(\mathrm{P}<0.001$; Fig. 2A). Increasing curcumin concentration resulted in increased cell death $(\mathrm{P}<0.001)$ in $2 \mathrm{D}$ monolayers, which was a behavior independent on the irradiation dose (Fig. 2B-E). However, in 3D spheroids the effect on cell viability was minimal and not proportional to irradiation dose (Fig. 2B-E).

TrxR1 concentration. The level of TrxR1 in 2 and 3D MCF-7 cell cultures treated with or without curcumin is shown in Fig. 3. The Trxs R1 level in 3D spheroids was significantly higher compared with that in 2D monolayers in untreated, curcumin (20 and $50 \mu \mathrm{M}$ ), FAC and radiation (6 and $8 \mathrm{~Gy}$ ) treated groups at both time intervals $(\mathrm{P}<0.001)$. After $24 \mathrm{~h}$ of treatment, 3D cultures showed a significant decrease in the TrxR1 level compared with that in the untreated cells at the 50 and $100 \mu \mathrm{M}$ curcumin dose $(\mathrm{P}<0.001)$. At a concentration of $100 \mu \mathrm{M}$ curcumin, 1.5- and 2.5 -fold reductions in TrxR1 levels were detected in the 2 and 3D cultures, respectively. After $48 \mathrm{~h}$ of treatment, no significant change in the TrxR1 level was observed in the 2D cultures. By contrast, a significant decrease was detected in 3D cultures with increasing curcumin dose. Chemotherapy led to a significant elevation in TrxR1 level in compared with the untreated 2 and 3D cultures at both time intervals $(\mathrm{P}=0.001)$. Radiation treatment at lower doses ( 2 and 4 Gy) caused a significant elevation in TrxR1 levels in 2 and 3D cultures compared with the untreated cells after $48 \mathrm{~h}$. This effect decreased with increasing radiation dose, whereby the TrxR1 level was significantly reduced compared with the untreated cells at $8 \mathrm{~Gy}$ in $2 \mathrm{D}$ culture after $24 \mathrm{~h}$ (Fig. 3). The TrxR1 level in cells treated with $100 \mu \mathrm{M}$ of curcumin was significantly lower compared with those treated with FAC in both 2 and 3D cultures at both time intervals $(\mathrm{P}<0.001$; Fig. 3 ). In the radiotherapy treated groups, $100 \mu \mathrm{M}$ curcumin-treated 2D and showed a significantly lower TrxR1 level compared to those treated with lower doses of radiation ( 2 and 4 Gy) at both time intervals (Fig. 3). However, at higher radiation doses (6 and 8 Gy), only 3D cultures showed a significant increase in TrxR1 levels compared to $100 \mu \mathrm{M}$ curcumin treated group ( $\mathrm{P}<0.001$; Fig. 3).

The combined treatment of chemotherapy and $100 \mu \mathrm{M}$ curcumin led to a significant reduction in TrxR1 level compared with that in the untreated group at all treatment intervals and both culture settings ( $\mathrm{P}<0.001$, Fig. 4A). Compared with that after chemotherapy, the combined treatment also led to a significant reduction of TrxR1 levels in 3D cultures in a dose-dependent manner at all time intervals. However, 2D culture only showed a significant reduction at FAC $+100 \mu \mathrm{M}$ Cur concentration $(\mathrm{P}<0.001$, Fig. 4A). Trx R1 levels were significantly higher in 3D spheroids compared with that in 2D monolayers at all treatment conditions and time intervals $(\mathrm{P}<0.001$, Fig. 4A). The combination of radiation and curcumin also led to a significant reduction in TrxR1 levels in a dose-dependent manner in all 3D culture settings compared to both control and groups treated with radiation alone ( $\mathrm{P}<0.001$; Fig. 4B-E). However, the 2D cultures didn't show a similar response unless the radiation doses was increased up to 6 and $8 \mathrm{~Gy}(\mathrm{P}<0.001$; Fig. $4 \mathrm{D}$ and $\mathrm{E})$. The 3D spheroids demonstrated significantly higher TrxR1 levels compared with that in the $2 \mathrm{D}$ monolayer group $(\mathrm{P}<0.001)$ and the difference was more prominent with higher radiation doses (Fig. 4B-E). 
A
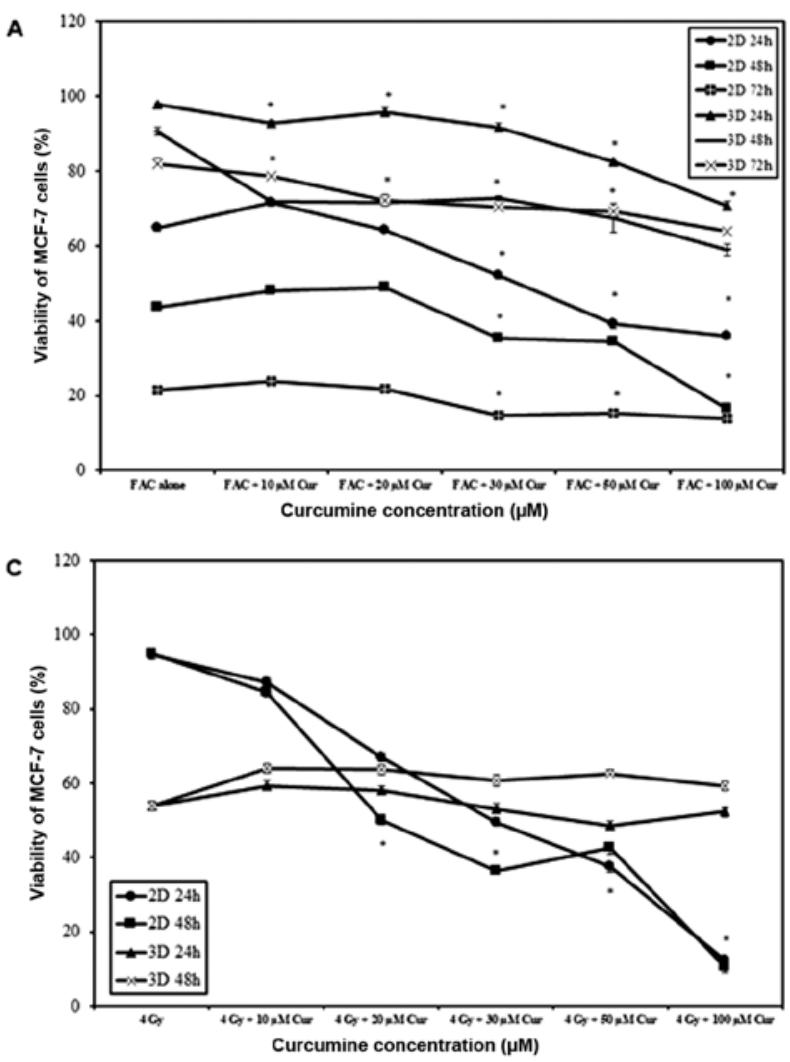

B

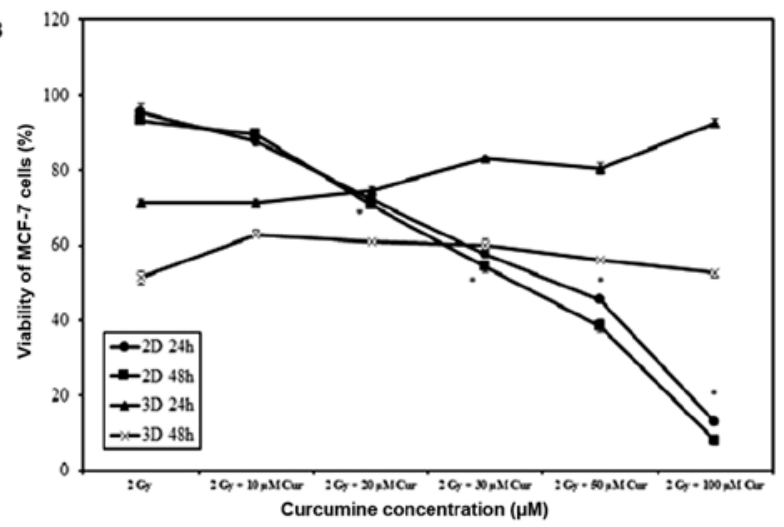

D

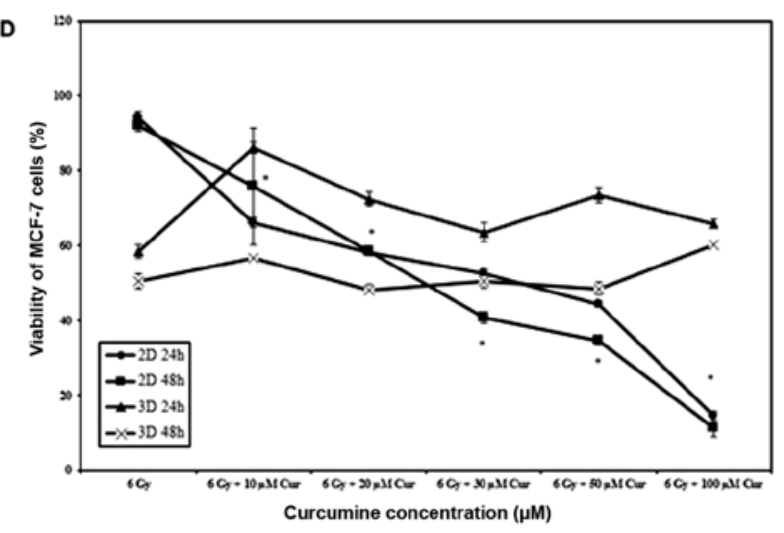

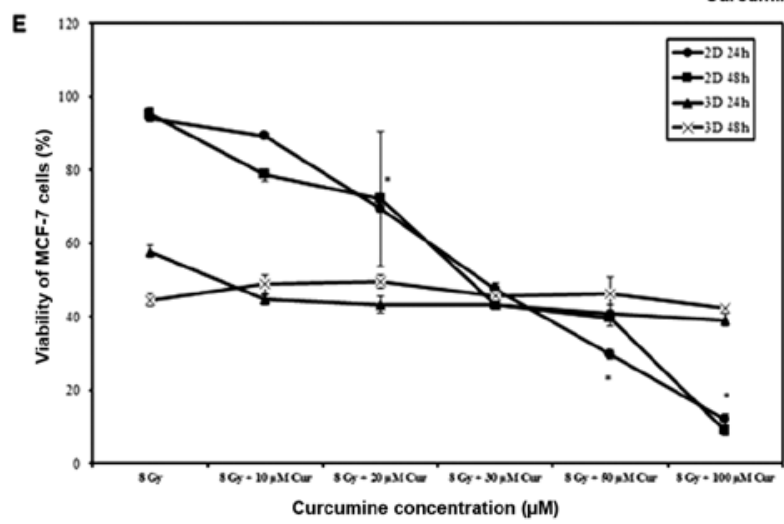

Figure 2. Viability of MCF-7 cells under different combinations of curcumin, chemotherapy and radiotherapy. MCF-7 cells were treated with different concentrations of curcumin combined either with (A) chemotherapy or radiotherapy at (B) 2, (C) 4, (D) 6 and (E) 8 Gy in 2 and 3D cultures. Results represent the $\%$ relative to the control and are presented as mean $\pm \mathrm{SD}(\mathrm{n}=5) .{ }^{*} \mathrm{P}<0.001$ vs. $100 \%$ viability of untreated cells. Cur, curcumin; FAC, 5-Fluorouracil-Adri amycin-cyclophosphamide.

Trx and TrxRl gene expression. The results of the Trx and TrxR1 gene expression assays in 2 and 3D cultures are presented in Fig. 5 as a fold-change of the control. In 2 and 3D cultures, treatment with $20 \mu \mathrm{M}$ curcumin led to a significant increase $(\mathrm{P}<0.001)$ in Trx and TrxR1 gene expression in compared with the control (Fig. 5A). At $100 \mu \mathrm{M}$ concentration, the expression of Trx and TrxR1 in 2D cultures was slightly increased, while in 3D cultures, it was significantly lower compared with the control.

In all culture settings, treatment with chemotherapy alone significantly increased Trx and TrxR1 expression compared with those in the control group (Fig. 5B). Furthermore, combination treatment with curcumin resulted in a significant reduction in $\operatorname{Trx}$ and $\operatorname{Trx} \mathrm{R} 1$ expression in compared with control, particularly with higher curcumin doses $(100 \mu \mathrm{M})$.

With radiation treatment, Trx and TrxR1 expression in all culture settings were significantly increased in compared with the control $(\mathrm{P}<0.001)$. Combining radiotherapy with curcumin led to a significant reduction in Trx and TrxR1 expression in both culture settings $(\mathrm{P}<0.001)$. However, the reduction in $2 \mathrm{D}$ was more prominent compared with that in $3 \mathrm{D}$ cultures $(\mathrm{P}<0.001$; Fig. 5).

Bcl2 and BAX gene expression. The results of the $\mathrm{Bcl} 2$ and BAX gene expression assays in 2 and $3 \mathrm{D}$ cultures are presented in Fig. 6 as a fold-change of the control. In 2 and 3D cultures, treatment with $100 \mu \mathrm{M}$ curcumin combined either with FAC or 8 Gy radiation led to a significant downregulation of $\mathrm{Bcl} 2$ genetic expression compared to the cells treated with $100 \mu \mathrm{M}$ curcumin alone $(\mathrm{P}<0.001)$. However, the reduction in $\mathrm{Bcl} 2$ expression was significantly lower in the $2 \mathrm{D}$ cultures compared with the 3D cultures.

Cells treated with curcumin showed significant upregulation of $\mathrm{BAX}$ in $2 \mathrm{D}$ cultures either alone or in combination 


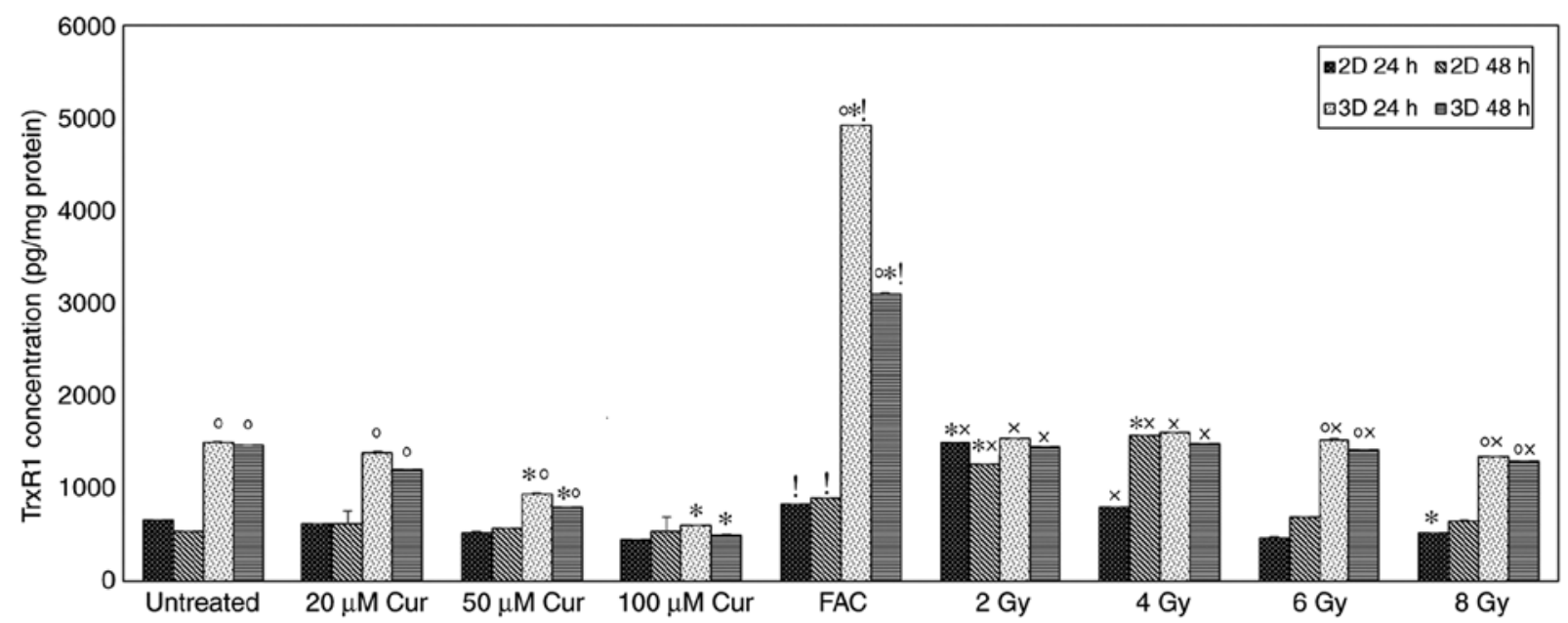

Figure 3. TrxR1 levels in MCF-7 cells treated with different concentrations of curcumin compared to standard treatments, FAC and radiation $(2,4,6$ and 10 Gy). Results are presented as mean $\pm \mathrm{SD}\left(\mathrm{n}=5\right.$ ). ${ }^{\prime} \mathrm{P}<0.001$ vs. untreated, ${ }^{\circ} \mathrm{P}<0.001$ vs. $2 \mathrm{D}$ culture, ${ }^{\mathrm{P}} \mathrm{P}<0.001$ vs. $100 \mu \mathrm{M}$ Cur, ${ }^{\times} \mathrm{P}<0.001$ vs. Cur (50 \& $100 \mu \mathrm{M}$ ) Cur, curcumin; FAC, 5-Fluorouracil-Adriamycin-cyclophosphamide; TrxR1, thioredoxin reductase.
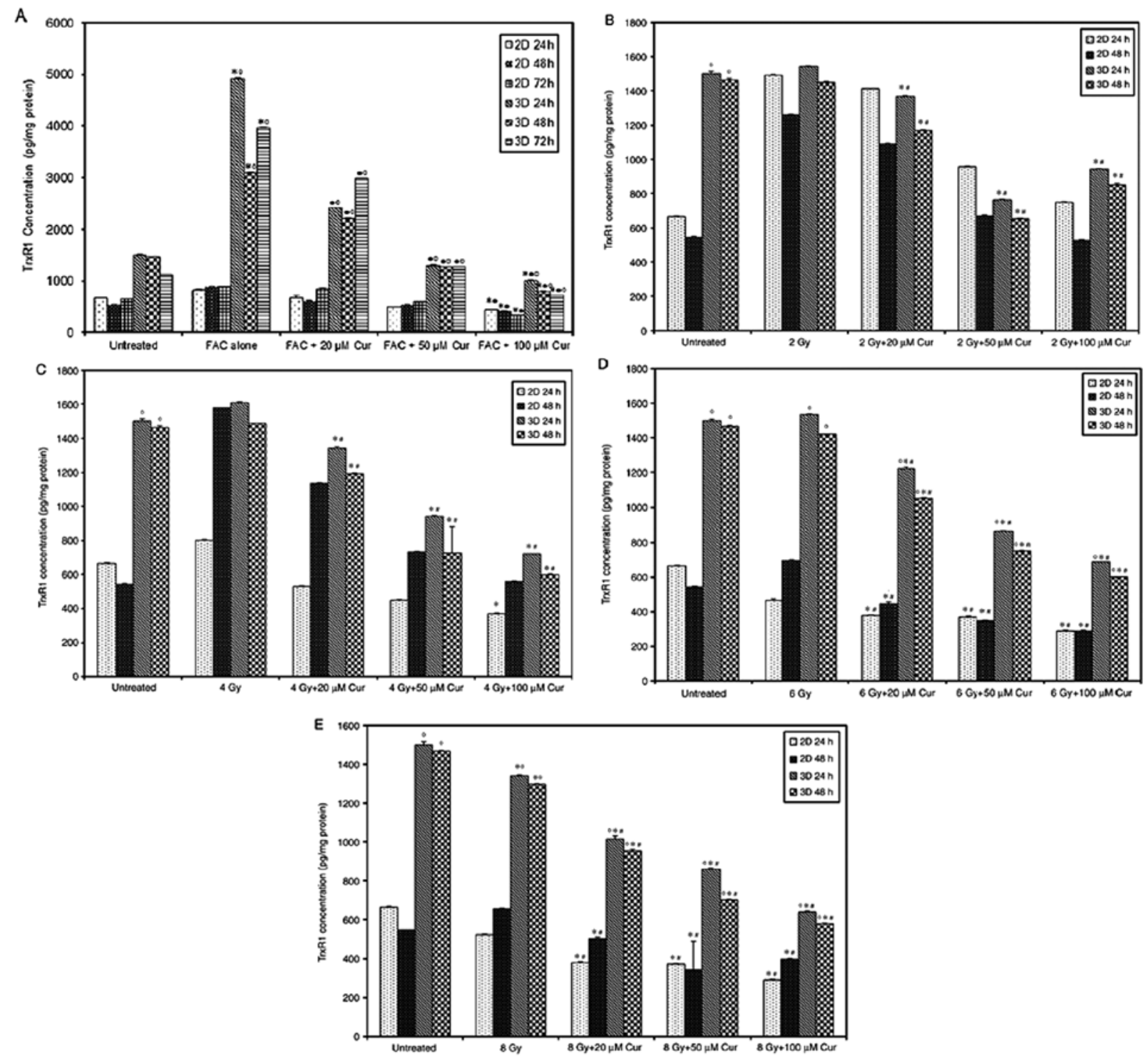

Figure 4. TrxR1 levels in MCF-7 cells in 2 and 3D cultures treated with curcumin combined with chemotherapy or radiotherapy. MCF-7 cells in 2 and 3D cultures treated with curcumin combined with (A) chemotherapy, (B) 2, (C) 4, (D) 6 and (E) 8 Gy radiotherapy. Results are presented as mean \pm SD (n=5). ${ }^{*} \mathrm{P}<0.001$ vs. untreated; ${ }^{\mathrm{P}} \mathrm{P}<0.001$ vs. FAC alone; ${ }^{\sharp} \mathrm{P}<0.001$ vs. $2,4,6$ or 8 Gy alone; and ${ }^{\circ} \mathrm{P}<0.001$ vs. $2 \mathrm{D}$ culture. Cur, curcumin; FAC, 5-Fluorouracil-Adriamy cin-cyclophosphamide; TrxR1, thioredoxin reductase. 

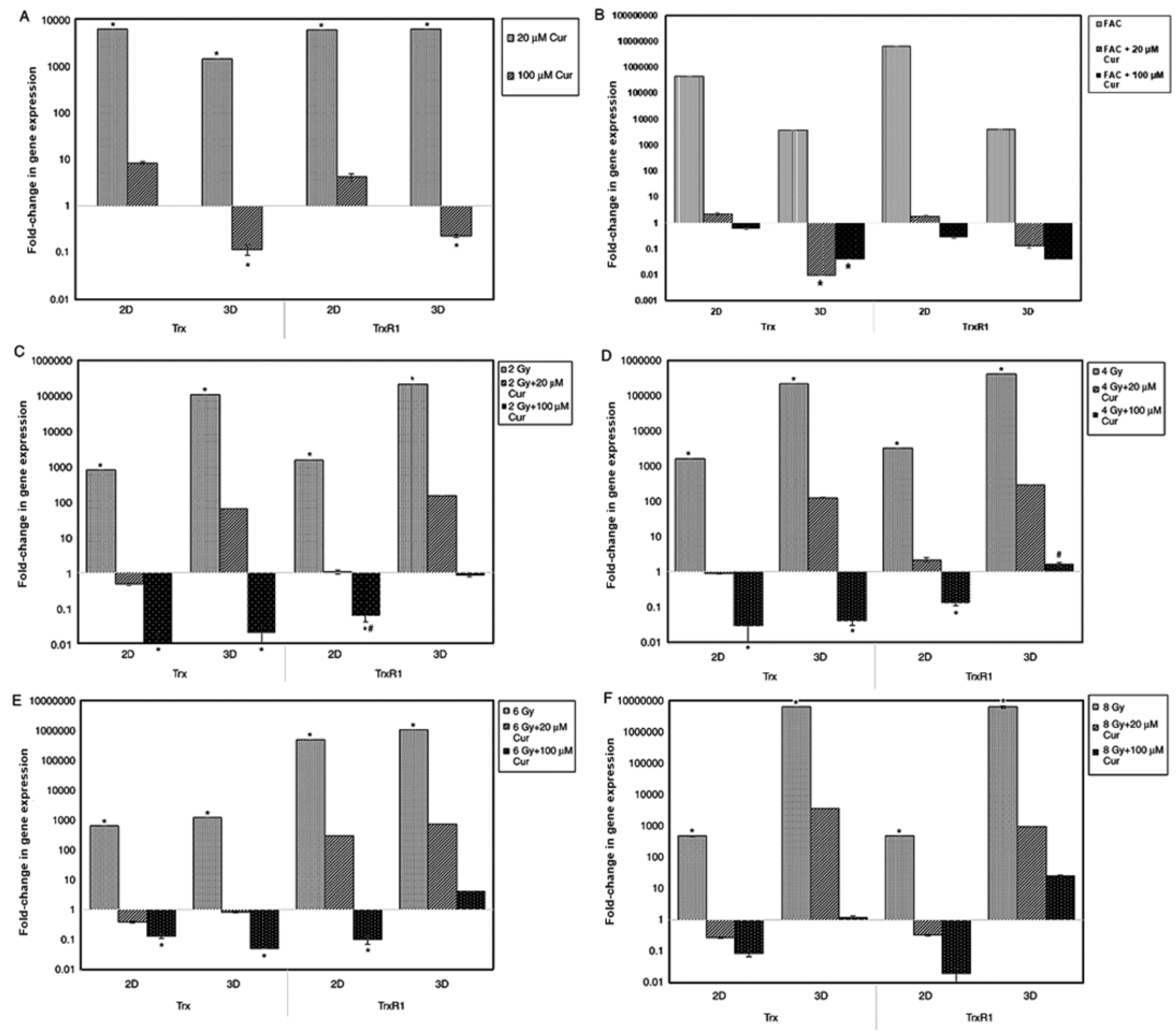

Figure 5. Expression of Trx and TrxR1 genes in 2 and 3D cultures of MCF-7 treated under different conditions. Expression of Trx and TrxR1 genes in 2 and 3D cultures of MCF-7 treated with (A) curcumin alone or combined with (B) chemotherapy, or (C) 2, (D) 4, (E) 6 and (F) 8 Gy radiotherapy for 48 h. Results are presented as mean $\pm \mathrm{SD}(\mathrm{n}=5)$. ${ }^{*} \mathrm{P}<0.001$ vs. control; ${ }^{\mathrm{P}} \mathrm{P}<0.0012 \mathrm{D}$ vs. 3D culture. Cur, curcumin; FAC, 5-Fluorouracil-Adriamycin-cyclophosphamide; Trx, thioredoxin; TrxR1, thioredoxin reductase.

with either FAC or 2-8 Gy radiation $(\mathrm{P}<0.001)$. Regarding the $3 \mathrm{D}$ cultures, the levels of BAX was significantly lower compared with the $2 \mathrm{D}$ cultures $(\mathrm{P}<0.001)$; however, treatment with curcumin + FAC or 8 Gy radiation lead to a significant upregulation in BAX gene levels.

\section{Discussion}

Targeting the $\operatorname{Trx} / \operatorname{TrxR}$ system inhibition is a promising approach for cancer treatment. Recently, many natural and synthetic compounds were developed to be used either alone or as adjuvants to existing cancer therapies, such as curcumin (8). In the current study, the use of curcumin as an anticancer/sensitizer agent was evaluated, through studying its effect on breast cancer (MCF-7) cell viability, TrxR1 activity and Trx/TrxR1 gene expression. MCF-7 2 and 3D cell culture systems were used, and cells were treated with different concentrations of curcumin alone or in combination with conventional treatment regimens, including chemo- and radiotherapy for different periods.
Curcumin significantly decreased MCF-7 cells viability in dose- and time-dependent manners in 2 and 3D systems. However, cell viability in 2D cultures was significantly lower compared to 3D cultures; most probably due to cell clustering effect that may prevent curcumin penetration to inner cells in spheroids. The results of the present study are in agreement with other studies in which a marked decrease in cell proliferation was associated with curcumin treatment in different types of cancer, including bladder (21), prostate (22), liver (23), and breast cancer (10). Curcumin function has been reported to be through p53-associated, caspase-dependent and mitochondrial mechanisms (24). Recently, Vallianou et al (25) proposed that curcumin can induce apoptosis through the induction of severe endoplasmic reticulum stress. In apoptosis-resistant cell lines, curcumin potentially activates cell death mechanisms, such as mitotic catastrophe, which is characterized by aberrant mitosis, multinucleated cells and giant cells (26).

The efficiency of chemotherapeutics is compromised by several metabolic and epigenetic alterations, and a constant 

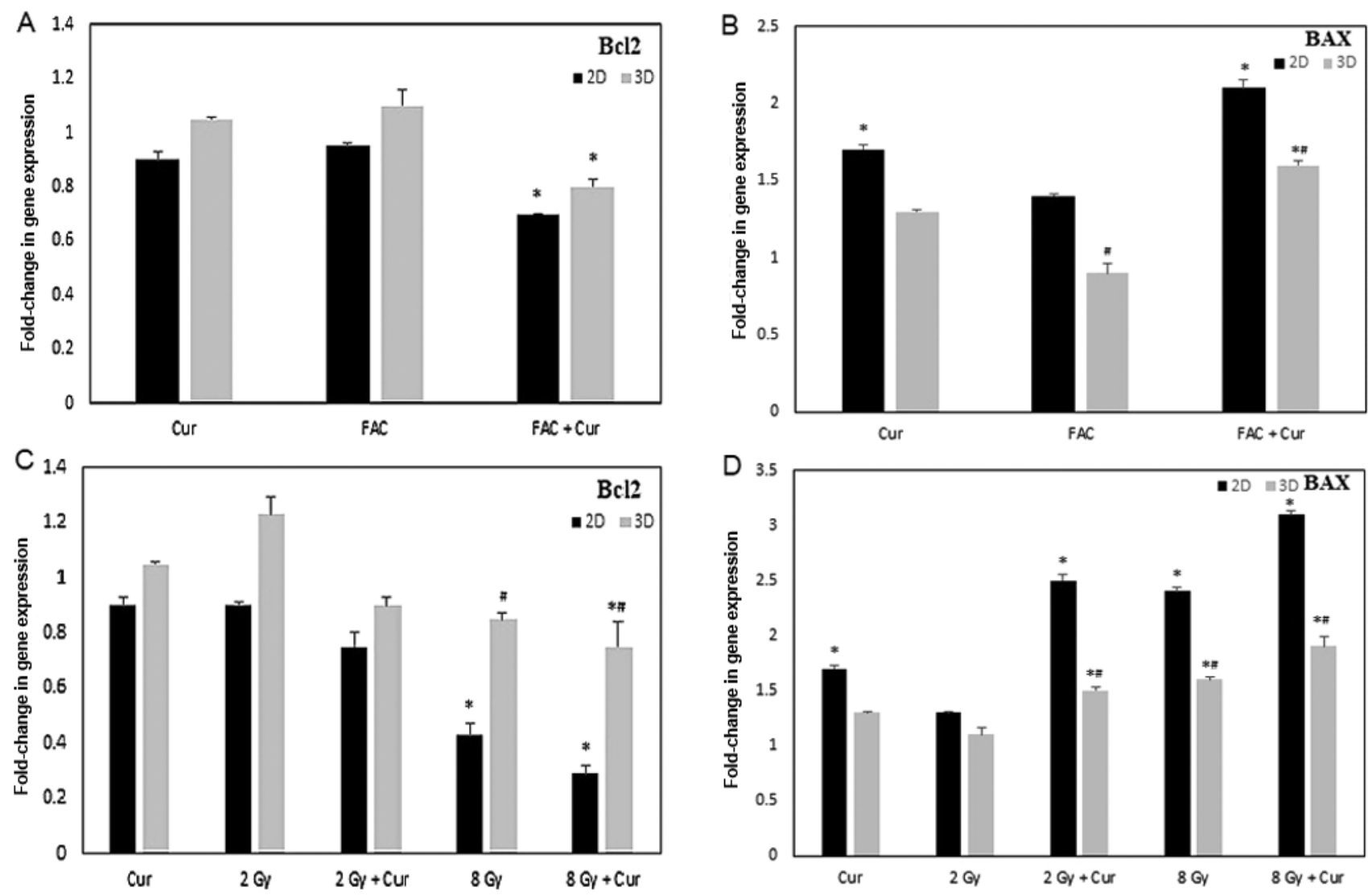

Figure 6. Expression of Bcl2 and BAX genes in 2 and 3D cultures of MCF-7 treated under different conditions. Expression of (A) Bcl2 and (B) BAX in cells treated with curcumin, FAC or combined treatment for $48 \mathrm{~h}$. Expression of (C) Bcl2 and (D) BAX in cells treated with 2 Gy, 8 Gy RAD either alone or combined with curcumin for $48 \mathrm{~h}$. Results are presented as mean $\pm \mathrm{SD}(\mathrm{n}=5){ }^{*} \mathrm{P}<0.001$ vs. control; ${ }^{*} \mathrm{P}<0.0012 \mathrm{D}$ vs. 3D culture. Cur, curcumin; FAC, 5-Fluorouracil-Adriamycin-cyclophosphamide.

shift in the tumor microenvironment leading to drug resistance (27). Although these results indicated that chemotherapy alone exhibited a substantial effect on MCF-7 cells in 2D cultures, the 3D culture showed a different response pattern to chemotherapy as no significant reductions in cell viability were observed after 24 or $48 \mathrm{~h}$, and cell viability reached $82 \%$ after $72 \mathrm{~h}$. The $2 \mathrm{D}$ cultures demonstrate maximum exposure to drugs and external stimuli in addition to limited cell-cell interaction, unlike 3D cultures that are multilayered with a hypoxic core, and show different exposure patterns to oxygen, nutrients and drugs. Hypoxic conditions in 3D spheroids resemble those observed in vivo in solid tumors, therefore, certain cytotoxic agents, such as doxorubicin, 5-FU or cisplatin, which work with oxygen, are less effective in 3D models $(28,29)$.

In the presence of curcumin with chemotherapy, the same pattern of effect between 2 and 3D cultures was noted in the present study. A significant reduction in cell viability and pro-apoptotic gene expression was observed in 2D cultures compared with in 3D cultures. In agreement with the results of the current study, Vinod et al (30) reported that curcumin works as a chemosensitizer for chemotherapy by silencing thymidylate synthase enzyme in breast cancer cells. Furthermore, Serasanambati et al (31), observed that $20 \mu \mathrm{M}$ of curcumin enhanced the antitumor effects of chemotherapy on MCF-7 breast cancer cells by decreasing cell proliferation (31). Additionally, Chen et al (32) reported that chemotherapy combined with curcumin showed modestly improved efficacy.
Regardless of the wide application of radiotherapy for breast cancer treatment, little is known about the response of 3D spheroids to radiation exposure. When exploring the effect of radiation on MCF-7 cell viability, 2D cultures showed little radiosensitivity in the present study. On the other hand, 3D cultures showed a significant reduction in cell viability in a time-dependent manner. By increasing radiation dose, the viability of $2 \mathrm{D}$ cultures did not show any significant change, whilst the viability of cells cultured in the 3D system showed a mild decrease. This is contradictory to other published reports that stated that cells cultured in a 3D system exhibit higher radioresistance levels compared with cells cultured in a $2 \mathrm{D}$ system $(33,34)$ These observations may be associated to the reduced thickness of monolayer cultured cells, which allows for little energy deposition within the cells. Meanwhile, the increased thickness of spheroids in 3D cultures may allow for higher energy deposition and subsequently an increased effect. In addition, cell-cell interactions may promote the response to radiotherapy through a bystander effect. El-Ashmawy et al (35) reported that irradiation of human lung epithelial 3D cell cultures showed reduced frequency of progression toward malignant phenotypes compared with 2D monolayers regardless of the comparable number of colonies (35).

Treatment of 2D monolayers with curcumin in combination with irradiation resulted in immediate radiosensitization in a time- and dose-dependent manner, which was the opposite to that observed on the $3 \mathrm{D}$ spheroids in which the effect was 
minimal. In line with the current results, the radiosensitizing effect of curcumin on 2D-radioresistant MCF-7 cells has been reported (36). Similar results were also reported in other types of cancer, including prostate cancer, musculoskeletal cancer (37), non-Hodgkin's lymphoma (38) and aggressive lymphomas (39). The discrepancy in culture viability may be explained by the downregulation of $\mathrm{Bcl} 2$ and upregulation of $\mathrm{BAX}$, which leads to a shift in the $\mathrm{Bcl} 2 / \mathrm{BAX}$ ratio towards a pro-apoptotic signal in curcumin-treated cells A previous study indicated that curcumin may counteract the radiation-induced anti-apoptotic signals, including $\mathrm{Bcl} 2$ elevation (40). Further investigations including Ki67/caspase-3 immunohistochemistry staining or flow cytometry would have been beneficial to back up these findings.

The effect of curcumin on cell viability was suspected to occur through Trx/TrxR1 modulation. TrxR1 is an oxidoreductase enzyme that serves a key role in maintaining redox-regulated cellular functions, including transcription, DNA damage recognition and repair, proliferation, and apoptosis (41). Cytosolic TrxR1 expression is often upregulated in human cancer, where it is associated with aggressive tumor growth and poor prognosis (42). The activity and ability of TrxR 1 to overcome oxidative stress induced in tumor cells via different treatment modalities depends on numerous factors, most importantly the availability of Trx (43).

The results of the current study demonstrated that TrxR1 concentrations in $3 \mathrm{D}$ cultures were significantly higher (approximately three times) compared with that in cells cultured in a 2D system; however, the levels significantly decreased upon curcumin treatment in a dose-dependent manner. At the transcriptional level, low curcumin concentration $(20 \mu \mathrm{M})$ resulted in a large induction of TrxR1 and $\operatorname{Tr} x$ genes, in 2 and 3D settings. Furthermore, high curcumin concentration $(100 \mu \mathrm{M})$ resulted in a mild induction of both genes in 2D cultures and significantly increased expression inhibition of both genes in 3D cultures. These results support the hypothesis of curcumin exhibiting dual functions. It has been postulated that curcumin may serve a protective role against oxidative stress at low concentrations and the opposite at high concentrations (44).

The different expression patterns of TrxR1 between 2 and $3 \mathrm{D}$ cultures have been reported previously in a colon cancer cell line. Lechner et al (45) reported that $>70 \%$ of HT-29 cells grown in a monolayer were positive for TrxR, while cells grown as spheroids or as tumors in mice, were negative for TrxR1. Nevertheless, to the best of our knowledge, the discrepancy in the effects of curcumin on TrxR1 concentration in 2D vs. 3D cultured cells were reported here for the first time. This observation reflects that cell-cell interactions, which are present in $3 \mathrm{D}$ cultured and are missing in 2D cultures, may impact the cellular response to therapeutic agents, such as curcumin.

It has been observed that cells overexpressing TrxR1 exhibit higher resistance to certain anticancer agents (46). The present work highlighted that cellular susceptibility to FAC is remarkably increased in curcumin-treated cells and hence enhanced cancer apoptosis. One possible explanation to rationalize this consequence may be the reduction of $\operatorname{Tr} \mathrm{R} 1$ via curcumin, which stimulates increasing ROS formation and consequently increases the cancer cell death rate (47). Similar results have also been reported for 5-FU, whereby curcumin was found to increase the sensitization of HCT116R cells to 5-FU-based chemotherapy (48). Although it is not clear whether the mechanism of sensitization involves TrxR1 or not (48). In agreement with the results of the current study, curcumin has been demonstrated to induce time-dependent ROS accumulation through suppression of TrxR1 activity and increase the level of the oxidized Trx form in cancer cells (49).

Upon exposure to radiotherapy, TrxR1 levels were elevated in 2 and 3D cultures following exposure to low doses of radiation; however, at higher radiation doses its levels were diminished in this study. The initial elevation in TrxR1 concentration is probably associated with high ROS generation by radiation in cell cytoplasm, which elicits an induced elevation of TrxR1 as an antioxidant defense mechanism. However, the subsequent reduction in $\operatorname{TrxR} 1$ concentration with increased radiation dose may be associated with increased apoptosis and/or impaired cellular responses resulting from radiation damage (14).

The present study possesses certain limitations. These limitations include the lack of western blotting technique to confirm the effect of curcumin on the translational level of TrxR1 and the lack of use of specific TrxR1 inhibitor for comparative studies. Morphological investigations should be analyzed to fully characterize the mechanisms underlying the potential involvement of the $\operatorname{Tr} x / \operatorname{Trx} \mathrm{R} 1$ system on the effects of curcumin on 2 and 3D MCF-7 cells.

In conclusion, the present findings showed that the cell culture setting has a significant effect on the cellular behavior and expression patterns, where the 3D model was shown to be a promising tool to represent tumors. The modulation of Trx/TrxR system expression suggests that it may be associated with increased cell death upon treatment with curcumin. These results also suggest that curcumin may have a potential role as a radiosensitizing and/or a chemosensitizing agent, providing a novel way to overcome cancer drug resistance.

\section{Acknowledgements}

Not applicable.

\section{Funding}

No funding was received.

\section{Availability of data and materials}

The datasets used and/or analyzed during the current study are available from the corresponding author on reasonable request.

\section{Authors' contributions}

SEEF: Sharing in study design and methodology, primary and additional laboratory analyses. MAGM: Sharing in conceptualization and manuscript revision and can authenticate the raw data. NAAEM: Determination of standard treatments' regimens, dose calculations and manuscript revision. ERZ: sharing in conceptualization, data analysis and interpretation and can authenticate the raw data. SAK: Co-participation in cell culture procedures, enzymatic protein quantification and molecular biology investigations. LMAA: Study design and methodology, primary and additional laboratory investigations. 
All authors have read and approved the final version of the manuscript.

\section{Ethics approval and consent to participate}

Not applicable.

\section{Patient consent for publication}

Not applicable.

\section{Competing interests}

The authors declare that they have no competing interests.

\section{References}

1. Bray F, Ferlay J, Soerjomataram I, Siegel RL, Torre LA and Jemal A: Global cancer statistics 2018: GLOBOCAN estimates of incidence and mortality worldwide for 36 cancers in 185 countries. CA Cancer J Clin 68: 394-424, 2018.

2. Hornsveld $M$ and Dansen TB: The hallmarks of cancer from a redox perspective. Antioxid Redox Signal 25: 300-325, 2016.

3. Lu J and Holmgren A: Thioredoxin system in cell death progression. Antioxid Redox Signal 17: 1738-1747, 2012.

4. Zhang J, Li X, Han X, Liu R and Fang J: Targeting the thioredoxin system for cancer therapy. Trends Pharmacol Sci 38: 794-808, 2017.

5. Haas B, Schütte L, Wos-Maganga M, Weickhardt S, Timmer M and Eckstein N: Thioredoxin confers intrinsic resistance to cytostatic drugs in human glioma cells. Int J Mol Sci 19: 2874, 2018.

6. Selenius M, Hedman M, Brodin D, Gandin V, Rigobello MP Flygare J, Marzano C, Bindoli A, Brodin O, Björnstedt M and Fernandes AP: Effects of redox modulation by inhibition of thioredoxin reductase on radiosensitivity and gene expression. J Cell Mol Med 16: 1593-1605, 2012.

7. Kim SJ, Miyoshi Y, Taguchi T, Tamaki Y, Nakamura H, Yodoi J, Kato K and Noguchi S: High thioredoxin expression is associated with resistance to docetaxel in primary breast cancer. Clin Cancer Res 11: 8425-8430, 2005.

8. Fang J, Lu J and Holmgren A: Thioredoxin reductase is irreversibly modified by curcumin: A novel molecular mechanism for its anticancer activity. J Biol Chem 280: 25284-25290, 2005.

9. Panda AK, Chakraborty D, Sarkar I, Khan T and Sa G: New insights into therapeutic activity and anticancer properties of curcumin. J Exp Pharmacol 9: 31-45, 2017.

10. Liu J, Pan Y, Chen O, Luan Y, Xue X, Zhao J, Liu L and Jia HY: Curcumin inhibits MCF-7 cells by modulating the NF- $\kappa$ B signaling pathway. Oncol Lett 14: 5581-5584, 2017.

11. Hewlings SJ and Kalman DS: Curcumin: A review of its' effects on human health. Foods 6: 92, 2017.

12. Zou J, Zhu L, Jiang X, Wang Y, Wang Y, Wang X and Chen B: Curcumin increases breast cancer cell sensitivity to cisplatin by decreasing FEN1 expression. Oncotarget 9: 11268-11278, 2018.

13. Javvadi P, Hertan L, Kosoff R, Datta T, Kolev J, Mick R, Tuttle SW and Koumenis C: Thioredoxin reductase- 1 mediates curcumin-induced radiosensitization of squamous carcinoma cells. Cancer Res 70: 1941-1950, 2010

14. Duval K, Grover H, Han LH, Mou Y, Pegoraro AF, Fredberg J and Chen Z: Modeling physiological events in $2 \mathrm{D}$ vs. 3D cell culture. Physiology (Bethesda) 32: 266-277, 2017.

15. Kapałczyńska M, Kolenda T, Przybyła W, Zajączkowska M, Teresiak A, Filas V, Ibbs M, Bliźniak R, Łuczewski $Ł$ and Lamperska K: 2D and 3D cell cultures-a comparison of different types of cancer cell cultures. Arch Med Sci 4: 910-919, 2018.

16. Knight E and Przyborski S: Advances in 3D cell culture technologies enabling tissue-like structures to be created in vitro J Anat 227: 746-756, 2015.

17. Vantangoli MM, Madnick SJ, Huse SM, Weston $P$ and Boekelheide K: MCF-7 human breast cancer cells form differentiated microtissues in scaffold-free hydrogels. PLoS One 10 e0135426, 2015

18. Imamura Y, Mukohara T, Shimono Y, Funakoshi Y, Chayahara N, Toyoda M, Kiyota N, Takao S, Kono S, Nakatsura T and Minami H: Comparison of 2D- and 3D-culture models as drug-testing platforms in breast cancer. Oncol Rep 33: 1837-1843, 2015.
19. Lowry OH, Rosebrough NJ, Farr AL and Randall RJ: Protein measurement with the Folin phenol reagent. J Biol Chem 193: 265-275, 1951.

20. Livak KJ and Schmittgen TD: Analysis of relative gene expression data using real-time quantitative PCR and the 2(-Delta Delta C(T)) method. Methods 25: 402-408, 2001.

21. Shi J, Zhang X, Shi T and Li H: Antitumor effects of curcumin in human bladder cancer in vitro. Oncol Lett 14: 1157-1161, 2017.

22. Teiten MH, Gaascht F, Cronauer M, Henry E, Dicato M and Diederich M: Anti-proliferative potential of curcumin in androgen-dependent prostate cancer cells occurs through modulation of the wingless signaling pathway. Int J Oncol 38: 603-611, 2011.

23. Wang J, Wang $\mathrm{C}$ and $\mathrm{Bu} \mathrm{G}$ : Curcumin inhibits the growth of liver cancer stem cells through the phosphatidylinositol 3-kinase/ protein kinase $\mathrm{B} / \mathrm{mammalian}$ target of rapamycin signaling pathway. Exp Ther Med 15: 3650-3658, 2018.

24. Perrone D, Ardito F, Giannatempo G, Dioguardi M, Troiano G, Lo Russo L, DE Lillo A, Laino L and Lo Muzio L: Biological and therapeutic activities, and anticancer properties of curcumin. Exp Ther Med 10: 1615-1623, 2015.

25. Vallianou NG, Evangelopoulos A, Schizas N and Kazazis C: Potential anticancer properties and mechanisms of action of curcumin. Anticancer Res 35: 645-651, 2015.

26. Salvioli S, Sikora E, Cooper EL and Franceschi C: Curcumin in cell death processes: A challenge for CAM of age-related pathologies. Evid Based Complement Alternat Med 4: 181-190, 2007.

27. Das T, Sa G, Saha B and Das K: Multifocal signal modulation therapy of cancer: Ancient weapon, modern targets. Mol Cell Biochem 336: 85-95, 2010.

28. Hoarau-Véchot J, Rafii A, Touboul C and Pasquier J: Halfway between 2D and animal models: Are 3D cultures the ideal tool to study cancer-microenvironment interactions? Int J Mol Sci 19: 181,2018

29. Lv D, Hu Z, Lu L, Lu H and Xu X: Three dimensional cell culture: A powerful tool in tumor research and drug discovery. Oncol Lett 14: 6999-7010; 2017.

30. Vinod BS, Antony J, Nair HH, Puliyappadamba VT, Saikia M, Narayanan SS, Bevin A and Anto RJ: Mechanistic evaluation of the signaling events regulating curcumin-mediated chemosensitization of breast cancer cells to 5-fluorouracil. Cell Death Dis 4: e505, 2013.

31. Serasanambati M, Chilakapati SR, Manikonda PK, Kanala JR and Chilakapati DR: Anticancer effects of brucine and gemcitabine combination in MCF-7 human breast cancer cells. Nat Prod Res 29: 484-490, 2015.

32. Chen S, Liang Q, Xie S, Liu E, Yu Z, Sun L, Shin MC, Lee SJ, $\mathrm{He} \mathrm{H}$ and Yang VC: Curcumin based combination therapy for anti-breast cancer: From in vitro drug screening to in vivo efficacy evaluation. Front Chem Sci Eng 10: 383-388, 2016.

33. Hehlgans S, Eke I, Storch K, Haase M, Baretton GB and Cordes N: Caveolin-1 mediated radioresistance of 3D grown pancreatic cancer cells. Radiother Oncol 92: 362-370, 2009.

34. Storch K, Eke I, Borgmann K, Krause M, Richter C, Becker K, Schrock E and Cordes N: Three-dimensional cell growth confers radioresistance by chromatin density modification. Cancer Res 70: 3925-3934, 2010.

35. El-Ashmawy M, Coquelin M, Luitel K, Batten K and Shay JW: Organotypic culture in three dimensions prevents radiation-induced transformation in human lung epithelial cells. Sci Rep 6: 31669, 2016.

36. Girdhani S, Ahmed MM and Mishra KP: Enhancement of gamma radiation-induced cytotoxicity of breast cancer cells by curcumin. Mol Cell Pharmacol 1: 208-217, 2009.

37. Peddada KV, Peddada KV, Shukla SK, Mishra A and Verma V: Role of curcumin in common musculoskeletal disorders: A review of current laboratory, translational, and clinical data. Orthop Surg 7: 222-231, 2015.

38. Qiao Q, Jiang Y and Li G: Curcumin enhances the response of non-Hodgkin's lymphoma cells to ionizing radiation through further induction of cell cycle arrest at the G2/M phase and inhibition of mTOR phosphorylation. Oncol Rep 29: 380-386, 2013.

39. Qiao Q, Jiang Y and Li G: Inhibition of the PI3K/AKT-NF- $\mathrm{B}$ pathway with curcumin enhanced radiation-induced apoptosis in human Burkitt's lymphoma. J Pharmacol Sci 121: 247-256, 2013.

40. Lia G, Wanga Z, Chonga T, Yangb J, Lia H and Chena $\mathrm{H}$ : Curcumin enhances the radiosensitivity of renal cancer cells by suppressing NF- $\mathrm{kB}$ signaling pathway. Biomed Pharmacother 94: 974-981, 2017.

41. Koháryová M and Kollárová M: Thioredoxin system-a novel therapeutic target. Gen Physiol Biophys 34: 221-233, 2015. 
42. Lu J and Holmgren A: The thioredoxin antioxidant system. Free Radic Biol Med 66: 75-87, 2014.

43. Stafford WC, Peng X, Olofsson MH, Zhang X, Luci DK, Lu L, Cheng Q, Trésaugues L, Dexheimer TS, Coussens NP, et al: Irreversible inhibition of cytosolic thioredoxin reductase 1 as a mechanistic basis for anticancer therapy. Sci Transl Med 10 eaaf7444, 2018.

44. Daverey A and Agrawal SK: Curcumin alleviates oxidative stress and mitochondrial dysfunction in astrocytes. Neuroscience 333: 92-103, 2016.

45. Lechner S, Müller-Ladner U, Neumann E, Spöttl T, Schlottmann K, Rüschoff J, Schölmerich J and Kullmann F: Thioredoxin reductase 1 expression in colon cancer: Discrepancy between in vitro and in vivo findings. Lab Invest 83: 1321-1331, 2003.

46. Koedrith P and Seo YR: Induction of doxorubicin-mediated apoptosis via thioredoxin reductase 1RNAi in human colon cancer cells. Mol Cell Toxicol 7: 112-119, 2011.
47. Eriksson SE, Prast-Nielsen S, Flaberg E, Szekely L and Arnér ES: High levels of thioredoxin reductase 1 modulate drug-specific cytotoxic efficacy. Free Radic Bio Med 47: 1661-1671, 2009.

48. Shakibaei M, Kraehe P, Popper B, Shayan P, Goel A and Buhrmann C: Curcumin potentiates antitumor activity of 5-fluorouracil in a 3D alginate tumor microenvironment of colorectal cancer. BMC Cancer 15: 205, 2015.

49. Shao FY, Du ZY, Ma DL, Chen WB, Fu WY, Ruan BB, Rui W, Zhang JX, Wang S, Wong NS, et al: B5, a thioredoxin reductase inhibitor, induces apoptosis in human cervical cancer cells by suppressing the thioredoxin system, disrupting mitochondrion-dependent pathways and triggering autophagy. Oncotarget 6: 30939-30956, 2015. 